\title{
DER DERZEITIGE UND DER ZUKÜNFTIGE KLIMAWANDEL IN DEN HISTORISCHEN PARKS BERLIN-BRANDENBURGS
}

\begin{abstract}
Um die Auswirkungen des Klimawandels auf historische Parkanlagen abzuschätzen, wurden beobachtete Klimadaten für die vier ausgewählte Parkanlagen in Berlin und Brandenburg ausgewertet und regionalen Modellsimulationen für zwei Zukunftsszenarien (RCP 4.5 und RCP 8.5) bis zum Jahr 2100 gegenüber gestellt. In der bodennahen Lufttemperatur erkennt man, dass im letzten Jahrhundert eine Erwärmung stattgefunden hat, die sich in der Zukunft fortsetzen wird (bis zum Jahr $2100 \mathrm{um} \mathrm{ca.} 1^{\circ} \mathrm{C}$ [RCP 4.5] bis $3^{\circ} \mathrm{C}$ [RCP 8.5]). Auch der Niederschlag hat in den letzten Jahrzehnten zugenommen, wobei der Winterniederschlag stärker anwuchs als der Sommerniederschlag. Dieser Trend setzt sich im RCP 8.5 Szenarium bis zum Jahr 2100 fort.
\end{abstract}

In order to estimate the impact of climate change on historic parks, climate data for four selected parks in Berlin and Brandenburg were analysed and compared with regional model simulations for two future scenarios (RCP 4.5 and RCP 8.5) up to the year 2100. In ground-level air temperature one can see that warming has occurred during the last century that will continue in future (up to the year 2100 by approximately $1^{\circ} \mathrm{C}$ [RCP 4.5] up to $3^{\circ} \mathrm{C}$ [RCP 8.5]). Rainfall has likewise increased in recent decades whereby winter precipitation increased more than summer precipitation. This trend will continue up to 2100 in the RCP 8.5 scenario.

\section{Einleitung}

Der Klimawandel hat zahlreiche Auswirkungen auf Natur und Umwelt, auch historische Parkanlagen sind davon betroffen. Die historischen Bedingungen in Berlin wurden ab

Im Text werden Maskulinum und Femininum verwendet, wenn es um Personen geht. Gemeint sind grundsätzlich alle Menschen, gleich welcher Geschlechtsidentität sie sich zugehörig fühlen.

Ә OpenAccess. ๑ 2019 Emmanuele Russo, Ulrich Cubasch und Gregor Pittke, Berlin-Brandenburgische Akademie der Wissenschaften, publiziert von De Gruyter. (c) BY-NC-SA Dieses Werk ist lizenziert unter der Creative Commons Attribution-NonCommercial-ShareAlike 4.0 Lizenz. https://doi.org/10.1515/9783110607482-014 
Gründung der Brandenburgischen Societät der Wissenschaften, der späteren KöniglichPreußischen Akademie der Wissenschaften (1711), ab dem Jahr 1700 (Cubasch/Kadow 2011a; Hupfer et al. 2013) aufgezeichnet. Weitere kürzere Beobachtungszeitreihen für Berlin wurden in Hupfer/Chmielewski (1990) sowie in der Berliner Klimafibel (2016) veröffentlicht. Die zukünftigen Änderungen des Berliner Klimas wurden für das IPCC-Szenarium Alb (»wir machen so weiter wie bisher«, Albritton et al. 2001) für den Zeitraum 2071-2100 basierend auf den Daten von Jacob et al. (2008) abgeschätzt (Cubasch/Kadow 2011b). Der bisherige und der voraussichtliche künftige Wandel des Brandenburger Klimas wurde in zwei umfangreichen Studien von Gerstengarbe et al. (2003) und Lotze-Campen et al. (2009) für den Zeitraum von 1951 bis 2055 analysiert, ebenfalls für das Szenarium A1b. In der hier vorliegenden Arbeit wird nun gezielt das Klima von vier historischen Parkanlagen im Bereich Berlin-Brandenburg untersucht.

\section{Daten}

Zur Abschätzung der bisherigen Bedingungen in den Parkanlagen wurden vier Messstationen des Deutschen Wetterdienstes ausgewählt, die nahe an den betrachteten Parkanlagen liegen:

Branitzer Park: Cottbus (ID: 00800)

Berlin, Tiergarten: Dahlem (ID: 00403)

Park Babelsberg: Potsdam (ID: 03987)

Wörlitzer Park: Wittenberg (ID: 05629)

Die hierfür verwendeten Daten wurden aus dem Datenarchiv des Deutschen Wetterdienstes gewonnen. Es muss beachtet werden, dass die einzelnen Standorte eine unterschiedliche Datenverfügbarkeit sowohl insgesamt als auch für verschiedene Parameter aufweisen (siehe Tabelle 1).

Um zukünftige Konditionen einzuschätzen und zu bewerten, wurden Klimasimulationen des EURO-CORDEX-Experiment-Frameworks (www.euro-cordex.net) ausgewertet.

Diese Simulationen basieren auf regionalen Klimamodellen, die mit Daten des Coupled Model Intercomparison Project Phase 5 (CMIP5) angetrieben wurden und eine Auflösung von $12 \mathrm{~km}$ besitzen. Um Unsicherheiten abzuschätzen, wurde ein Ensemble von drei Modellen betrachtet und verglichen. Es wurden zwei Emissionsszenarien (Representative Concentration Pathways - RCP; Stocker et al. 2013) gewählt, um die Auswirkung verschiedener Treibhausgaskonzentrationen auf die zukünftigen Klimabedingungen zu bestimmen. Das erste Szenarium (RCP 4.5) beschreibt steigende Treibhausgasemissionen, die bis 2040 ihr Maximum erreichen und danach abnehmen. Das zweite Szenario (RCP 8.5) beschreibt stetig steigende Emissionen bis Ende des 21. Jahrhunderts. Dieses Szenarium entspricht in etwa dem älteren Szenarium A1b (»wir machen so weiter wie bisher «). Vergleichsweise würde die mittlere globale Oberflächentemperatur im Jahr 2100 für RCP 4,5 um knapp 


\begin{tabular}{l|l|l|l}
\hline Station & \multicolumn{2}{l}{ Datenverfügbarkeit (von ... bis) } & Anmerkungen \\
\hline $\begin{array}{l}\text { Temperatur } \\
\text { Cottbus }\end{array}$ & 01.01 .1956 & 31.12 .2015 & $\begin{array}{l}\text { Datenlücken zwischen 1980 und 1983, } \\
1956 \text { weist viele Datenlücken innerhalb } \\
\text { des Jahres auf } \rightarrow \text { nicht betrachtet }\end{array}$ \\
\hline Dahlem & 01.01 .2002 & 31.12 .2015 & \\
\hline Potsdam & 01.01 .1893 & 31.12 .2015 & \\
\hline Wittenberg & 01.01 .1951 & 31.12 .2015 & Datenlücken zwischen 1974 und 1976 \\
\hline Niederschlag & & & \\
\hline Cottbus & 01.09 .1995 & 31.12 .2015 & \\
\hline Dahlem & 28.01 .2002 & 31.12 .2015 & \\
\hline Potsdam & 01.09 .1995 & 31.12 .2015 & \\
\hline Wittenberg & 01.09 .1951 & 31.12 .2015 & \\
\hline
\end{tabular}

Tabelle 1 Datenverfügbarkeit der einzelnen Messstandorte für die Parameter »2-Meter-Temperatur« und »Niederschlag«. Die Messstandorte registrieren auch über 2015 hinaus die betrachteten Parameter.

$2{ }^{\circ} \mathrm{C}$, für RCP 8,5 um über $4^{\circ} \mathrm{C}$ über den gegenwärtigen Werten liegen (vgl. Stocker et al. 2013, IPCC 2014).

Eine Liste der verwendeten Modellexperimente findet sich im Anhang (Datenquellen).

Folgende Parameter wurden untersucht:

1. Jährliche und saisonale Mittel der bodennahen Lufttemperatur (in 2 m Höhe), im Text allgemein als »Temperatur« bezeichnet

2. Saisonale Mittel der Maximumtemperatur

3. Saisonale Mittel der Minimumtemperatur

4. Anzahl der Tage mit Temperaturextremen

5. Jährliche und saisonale Niederschlagssummen

6. Jährliche und monatliche Mittel des Standardisierten Niederschlagsindexes (SPI)

\section{Ergebnisse}

\subsection{Temperatur}

Das bekannteste Merkmal des derzeitigen Klimawandels ist eine allgemein beobachtbare globale Erwärmung (Stocker et al. 2013). Unsere Analysen zeigen ebenfalls positive Trends der mittleren bodennahen Luftemperatur für die betrachteten Parkanlagen. 

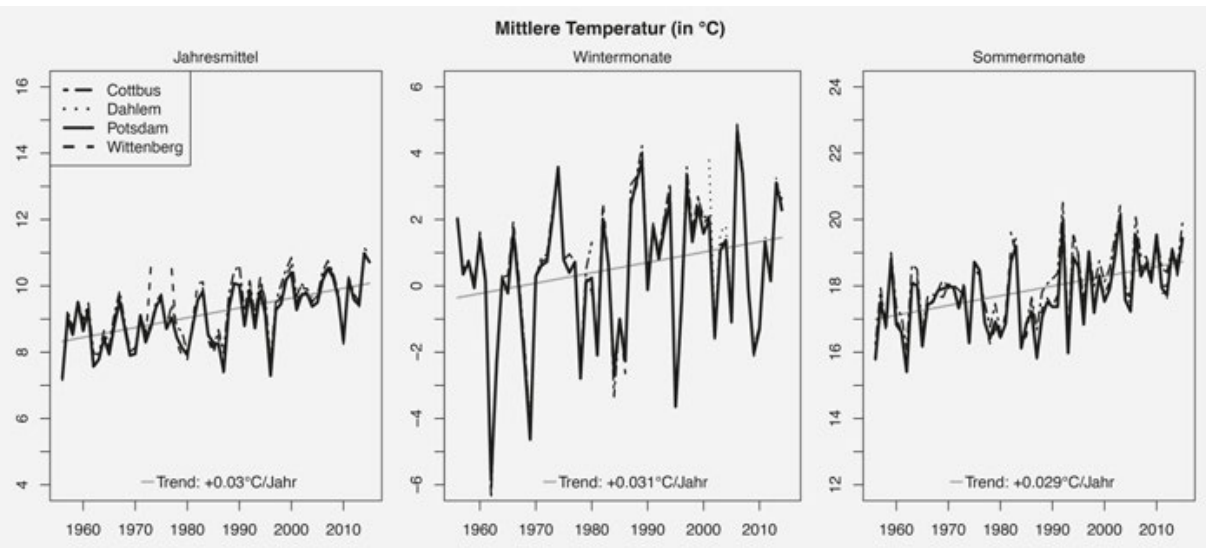

1 Die beobachteten Mitteltemperaturen an den Messstandorten und dazugehöriger Temperaturtrend im Zeitraum zwischen 1956 und 2015. Links: Jahresmitteltemperatur, Mitte: saisonales Mittel der Wintermonate, rechts: saisonales Mittel der Sommermonate.

Sowohl die jährliche als auch die jahreszeitliche Analyse zeigt hohe Übereinstimmungen zwischen den Beobachtungen an den einzelnen Messstandorten mit einem positiven Trend der Temperatur von $0,03^{\circ} \mathrm{C}$ pro Jahr (1951-2015), der deutlich über dem globalen Mittelwert von ca. $0,01^{\circ} \mathrm{C}$ pro Jahr liegt (vgl. Stocker et al. 2013; IPCC 2014).

Dieser positive Trend ist in den Frühlings- und Sommermonaten etwas schwächer ausgeprägt als in den Herbst- und Wintermonaten (Abb. 1).

Ein Vergleich der Temperaturmessungen mit den Modellergebnissen für den Zeitraum 1951 bis 2015 zeigt eine gute Übereinstimmung, wobei die Beobachtungsdaten überwiegend im Bereich der Modellstreuung lagen. Dies gilt auch für die aus den Daten abgeleiteten Trends.

Für die zukünftige Entwicklung bis zum Jahr 2100 zeigen die Modellergebnisse des RCP-4.5- und des RCP-8.5-Szenariums sowohl im jährlichen als auch im saisonalen Mittel einen signifikanten Anstieg der Mitteltemperatur, der beim zweiten Szenario stärker ausgeprägt ist. Die Mitteltemperatur der Emissionsszenarien liegt zum Ende des Jahrhunderts zwischen ca. $1^{\circ} \mathrm{C}$ (RCP 4.5) und $3^{\circ} \mathrm{C}$ (RCP 8.5) über dem Mittel des Zeitraumes 2006-2015 (Abb. 2). Dieses entspricht in etwa den Ergebnissen von Gerstengarbe et al. (2003) und Lotze-Campen et al. (2009), die in ihren Untersuchungen für Brandenburg einen Temperaturanstieg von ca. $1,4^{\circ} \mathrm{C}$ bis $3,1^{\circ} \mathrm{C}$ bis zum Jahr 2050 prognostizieren. Nach den genannten Ergebnissen würde der Temperaturanstieg im Untersuchungsgebiet für die betrachteten Szenarien niedriger als im globalen Maßstab (IPCC 2014) ausfallen.

Die Maximumtemperatur in den Beobachtungsdaten zeigt, ähnlich der Mitteltemperatur, hohe Übereinstimmung zwischen den einzelnen Standorten und einen positiven Trend, der sowohl jährlich als auch saisonal stark ausgeprägt ist (Abb. 3). 


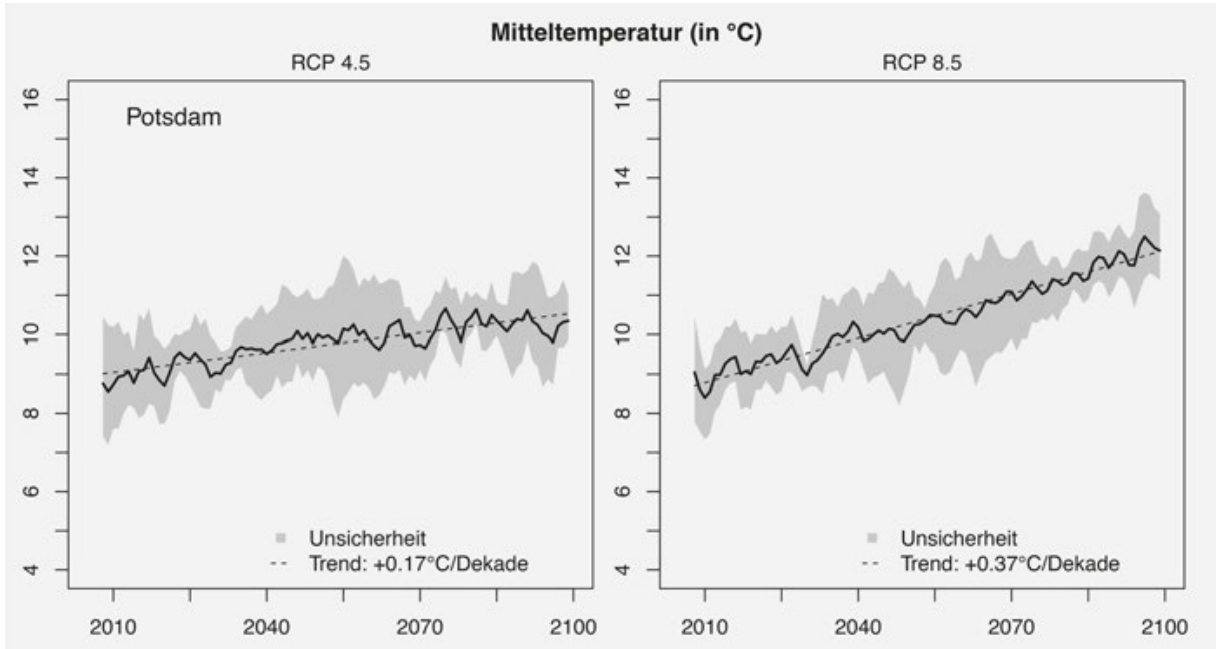

2 Projektion der jährlichen Mitteltemperatur für Potsdam im Zeitraum zwischen 2006 und 2100. Die grau schattierten Flächen beschreiben die Modellunsicherheit, die durchgezogene Linie das Mittel der Modelle. Die gestrichelte Linie zeigt den abgeleiteten Trend.

Ein Vergleich der Beobachtungen mit den Modellsimulationen (nicht dargestellt) zeigt im Zeitraum zwischen 2001 und 2015 nur geringe Abweichungen, jedoch werden die Temperaturspitzen vom Modell unterschätzt.

In den Zukunftsszenarien erkennt man (im Zuge der Fortsetzung der bereits stattgefundenen Erwärmung) eine Zunahme der Maximaltemperatur, die erwartungsgemäß im zweiten Emissionsszenarium stärker ausgeprägt ist als im ersten (Abb. 4). Dies deckt sich mit den Ergebnissen von Gerstengarbe et al. (2003) und Lotze-Campen et al. (2009), die für Brandenburg ebenfalls einen Anstieg der Temperatur und eine Zunahme der Tage mit extremen Temperaturen vorhersagen.

Da in der Vegetationsperiode auftretende Fröste ein Risiko für Pflanzen darstellen, die wiederum jedoch über die Winterzeit ein gewisses Maß an Kälte für die Winterruhe benötigen, werden die Minimumtemperatur und die Anzahl der Tage mit einer Minimumtemperatur unter $-5^{\circ} \mathrm{C}$ untersucht. In dieser Untersuchungen werden die Tage mit einer Minimumtemperatur unter $-5^{\circ} \mathrm{C}$ als Frosttage bezeichnet, weil dieser Temperaturgrenzwert für das Überleben der Pflanzen wichtiger ist als die $0{ }^{\circ} \mathrm{C}$-Grenze. Dieser Grenzwert entspricht nicht der meteorologischen Definition, ${ }^{1}$ bei der als Frosttage all jene Tage gelten, an denen die Minimumtemperatur $0^{\circ} \mathrm{C}$ unterschreitet. Für die Minimumtemperatur und die Frosttage erkennt man eine enge Übereinstimmung der Beobachtungen an den verschiedenen

1 Siehe DWD Lexikon: Frosttag, https://www.dwd.de/DE/service/lexikon/Functions/glossar.html?nn=103 $346 \& \operatorname{lv} 2=100784 \& \operatorname{lv} 3=100912$ (07.01.2019). 


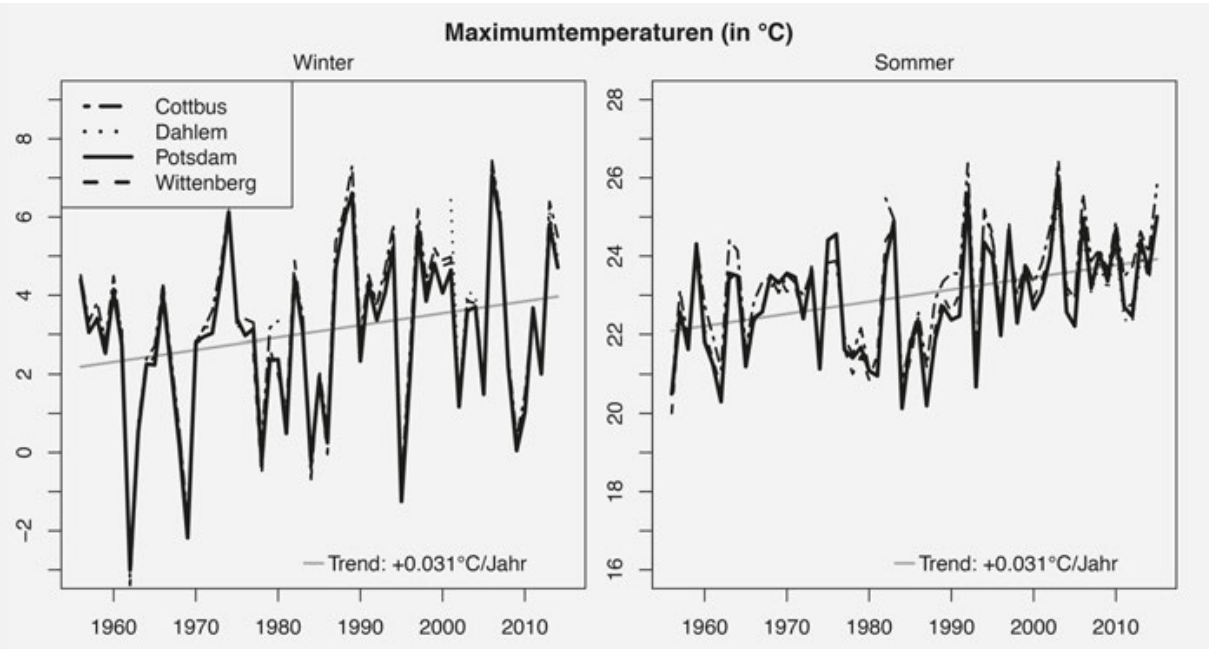

3 Saisonales Mittel der beobachteten Maximumtemperatur an allen Messstandorten im Zeitraum zwischen 1956 und 2015. Links für die Wintermonate (Dezember, Januar, Februar), rechts für die Sommermonate (Juni, Juli, August).

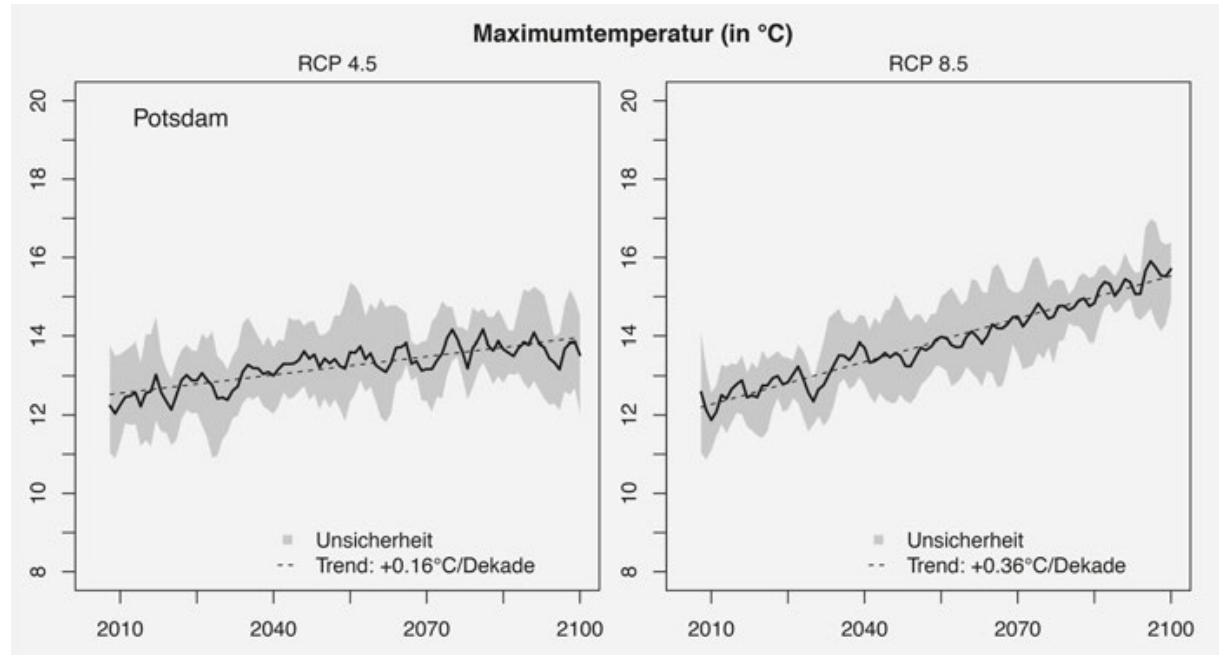

4 Projektion der jährlichen Maximumtemperatur am Beispiel Potsdam (schwarze Linie) im Zeitraum zwischen 2006 und 2100. Die schattierten Flächen beschreiben die Modellunsicherheit, die gestrichelte Linie zeigt den Temperaturtrend. 


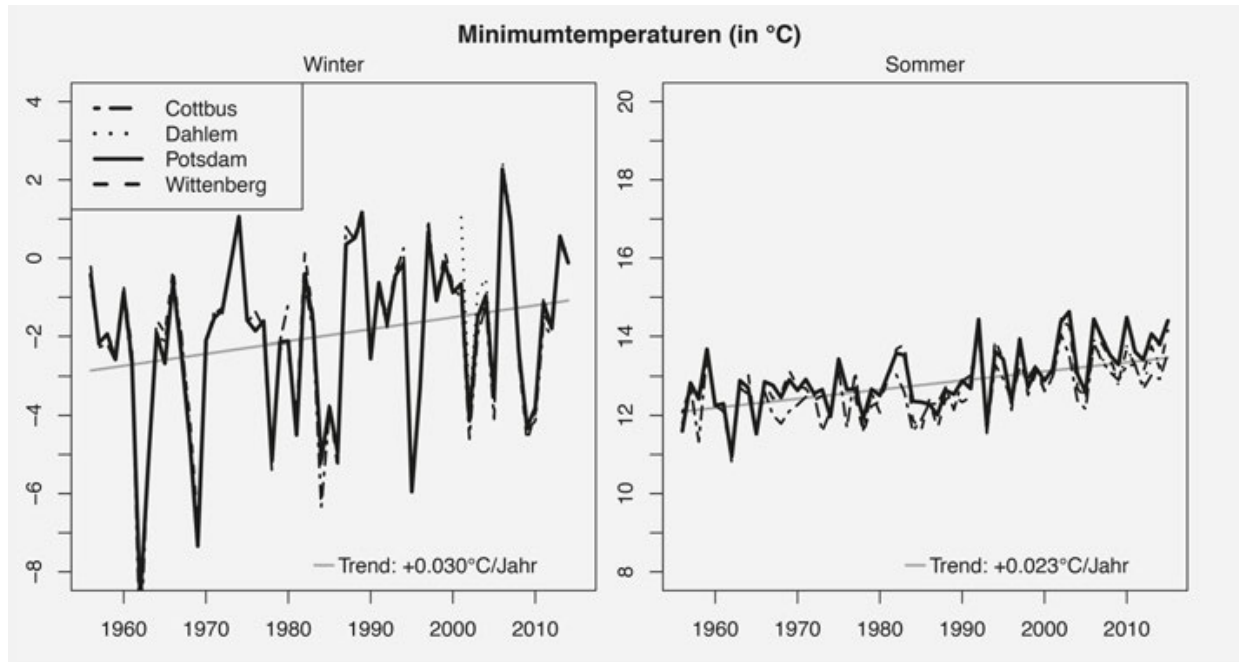

5 Mittel der beobachteten Minimumtemperatur an allen Messstandorten im Zeitraum zwischen 1956 und 2015. Links: saisonales Mittel der Wintermonate, rechts: saisonales Mittel der Sommermonate.

Standorten mit den Modellsimulationen (nicht abgebildet). Die Minimumtemperatur (Abb. 5) zeigt einen positiven Trend, der die bereits stattgefundene Erwärmung widerspiegelt. Entsprechend kann man für die Anzahl der Frosttage einen negativen Trend erkennen, also eine Abnahme der Anzahl der Frosttage.

Die Analyse der Projektionen zeigt einen fortlaufenden Trend der Erwärmung, der sich in beiden Szenarien in den positiven Trends der Minimumtemperatur bzw. den negativen Trends der Anzahl von Frosttagen widerspiegelt. Auch hier kann man einen stärker ausgeprägten Trend für das Emissionsszenarium mit höherem Treibhausgaskonzentrationsanstieg feststellen (Abb. 6). Für das weniger progressive Szenarium erkennt man gegen Ende der Projektionszeit eine Abschwächung des Trends, was die Stabilisierung der Treibhausgaskonzentrationen in diesem reflektiert.

Die Veränderung der Temperaturextreme in den Beobachtungen und in den Modellen lassen sich Tabelle 2 entnehmen. Die Modelle simulieren für 1981 bis 2005 eine ähnliche Anzahl an Eistagen (Tagestemperatur unter $0^{\circ} \mathrm{C}$ ) sowie Frosttagen (Tageshöchsttemperatur unter $-5^{\circ} \mathrm{C}$ ) wie beobachtet. Die Anzahl der Frosttage und der Eistage wird für die Periode 2076 bis 2100 bei dem Szenarium RCP 4.5 um ca. $40 \%$ vermindert, beim Szenarium 8.5 um $70 \%$. Die Modelle unterschätzen deutlich die Anzahl der Sommertage (Tageshöchsttemperatur gleich oder über $25^{\circ} \mathrm{C}$ ) sowie der Hitzetage (Tageshöchsttemperatur gleich oder über $30^{\circ} \mathrm{C}$ ). Das lässt auf eine unzureichende Darstellung oder das Fehlen von wichtigen Rückkopplungsprozessen insbesondere bei sehr extremen Erwärmungsereignissen in den Modellen schließen. Betrachtet man die relative Änderung der Sommer- und Hitzetage in den Modellen vom Zeitraum 1981-2005 zu 2076-2100, so erhält man für das 


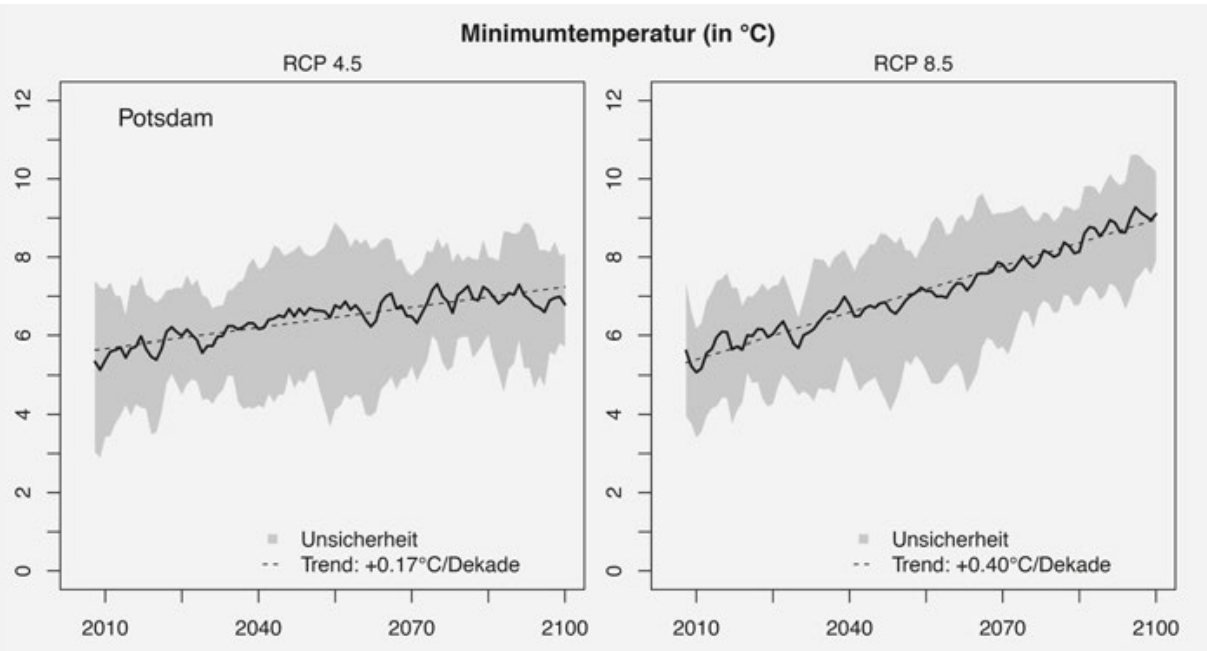

6 Projektion der jährlichen Minimumtemperatur am Beispiel Potsdam im Zeitraum zwischen 2006 und 2100. Die grau schattierten Flächen beschreiben die Modellstreuung, die durchgezogene Linie den Temperaturverlauf, die gestrichelte Linie zeigt den Trend. Die Projektion verläuft an den anderen Standorten nahezu identisch.

\begin{tabular}{|c|c|c|c|c|c|c|c|}
\hline \multicolumn{8}{|c|}{ historisch (1981-2005) } \\
\hline \multicolumn{2}{|c|}{$\begin{array}{l}\text { Eistage } \\
\left(\mathrm{T}_{\max }<0^{\circ} \mathrm{C}\right)\end{array}$} & \multicolumn{2}{|c|}{$\begin{array}{l}\text { Frosttage } \\
\left(\mathrm{T}_{\min }<-5^{\circ} \mathrm{C}\right)\end{array}$} & \multicolumn{2}{|c|}{$\begin{array}{l}\text { Sommertage } \\
\left(\mathrm{T}_{\max } \geq 25^{\circ} \mathrm{C}\right)\end{array}$} & \multicolumn{2}{|c|}{$\begin{array}{l}\text { Hitzetage } \\
\left(\mathrm{T}_{\max } \geq 30^{\circ} \mathrm{C}\right)\end{array}$} \\
\hline Obs. & Modelle & Obs. & Modelle & Obs. & Modelle & Obs. & Modelle \\
\hline 21,3 & 24,2 & 23,7 & 24,9 & 41,2 & 20,9 & 8,5 & 2,3 \\
\hline \multicolumn{8}{|c|}{ zukünftig (2076-2100) } \\
\hline RCP 4.5 & RCP 8.5 & RCP 4.5 & RCP 8.5 & RCP 4.5 & RCP 8.5 & RCP 4.5 & RCP 8.5 \\
\hline 15,9 & 7,0 & 14,9 & 6,6 & 30,4 & 44,6 & 4,8 & 9,9 \\
\hline
\end{tabular}

Tabelle 2 Mittlere Anzahl von Temperaturextremen pro Jahr in einem 25-jährigen historischen und zukünftigen Zeitraum. Beobachtungsdaten (Obs.) und Modelldaten (Modelle) jeweils Stations- bzw. Gitterpunktmittel

RCP 4.5 Szenarium ca. $50 \%$ mehr Sommertage und ca. $100 \%$ mehr Hitzetage, bei Szenarium RCP 8.5 verdoppelt sich die Anzahl der Sommertage und vervierfacht sich die Zahl der Hitzetage.

\subsection{Niederschlag}

In den Beobachtungen zeigt der mittlere Niederschlag vergleichbare Werte an allen Standorten, wobei die Variabilität in den Sommermonaten wegen des vermehrten Auftretens konvektiver Ereignisse stärker ist. Insgesamt verzeichnet man in den vergangenen Jahren 

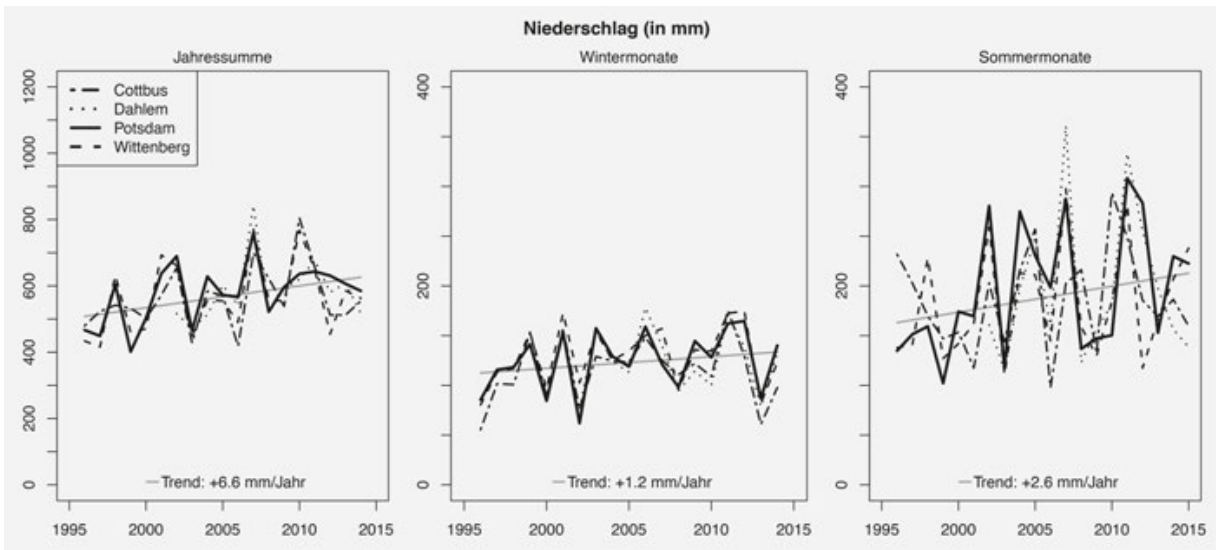

7 Jahressumme des Niederschlags an allen Standorten im Zeitraum zwischen 1996 und 2015. Links: Jährlicher Gesamtniederschlag, Mitte: Niederschlagsmenge in den Wintermonaten, rechts: Niederschlagssumme in den Sommermonaten.

eine leichte Niederschlagszunahme, die in den Sommermonaten stärker ausgeprägt ist als in den Wintermonaten (Abb. 7).

Ein Vergleich unserer Modellergebnisse mit den Beobachtungen zeigt große Unterschiede (nicht dargestellt), was auf die bekannte Problematik der Niederschlagssimulationen auf kleinen Skalen hindeutet (Stocker et al. 2013). Man stellt eine Überschätzung des Niederschlags durch das Modell besonders bei geringen Niederschlagsmengen fest.

Wegen dieser Unsicherheiten in den Niederschlagssimulationen wird in der weiteren Analyse nicht der Niederschlag direkt betrachtet, sondern der Standardisierte Niederschlagsindex SPI (Standardized Precipitation Index; Hayes/Lowrey 2007), der über eine statistische Normalisierung der Niederschlagsdaten eine stabile niederschlagsbezogene Information erzeugt.

Er wird routinemäßig eingesetzt, um Dürren und Trockenperioden zu identifizieren und den dadurch verursachten Trockenstress und den Bedarf an künstlicher Bewässerung abzuschätzen. Er eignet sich besonders für die Analyse monatlicher Niederschläge, weshalb hier statt saisonaler monatliche Untersuchungen angestellt werden. Der Index gibt die Anzahl an Standardabweichungen an, um die das aufsummierte Niederschlagsdefizit des momentan betrachteten Wertes vom normalisierten Mittel abweicht. Einen kurzen Abriss über diesen häufig verwendeten Index findet man auf der Webseite des Deutschen Wetterdienstes. ${ }^{2}$

Zur Berechnung des SPIs wurden in diesem Bericht die Beobachtungsdaten und die historischen Modelldaten verwendet. Der SPI lässt sich in Kategorien einteilen, die unterschiedliche Schweregrade von Trockenheit bzw. Feuchtigkeit beschreiben (siehe Tabelle 3; Hayes/Lowrey 2007). 

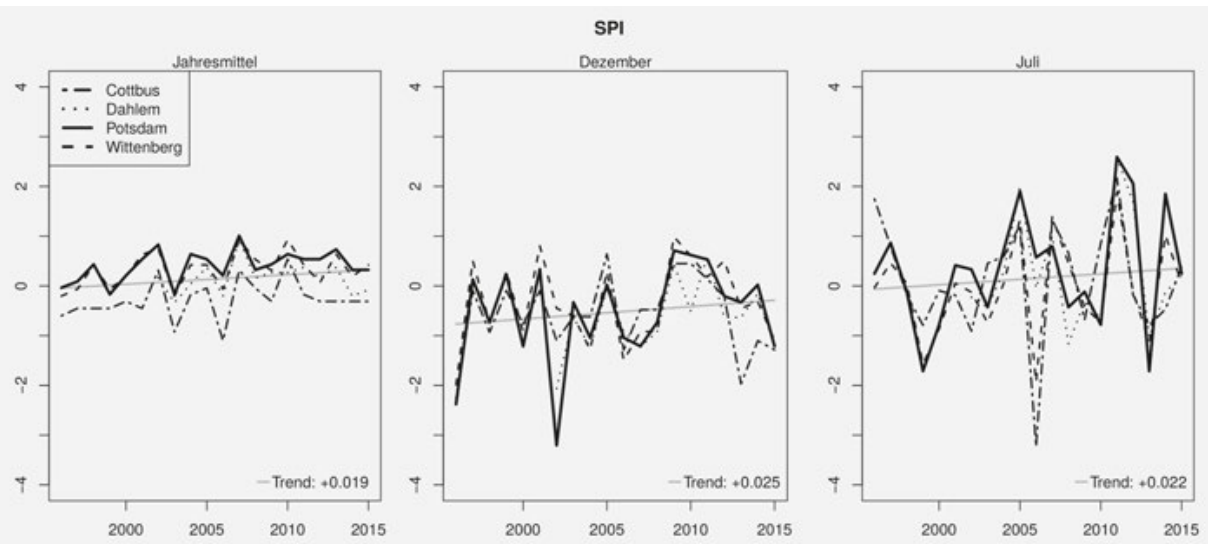

8 Der SPI, berechnet aus Beobachtungen an allen Standorten für den Zeitraum zwischen 1951 und 2015. Die durchgezogene Linie gibt den Mittelwert an, die gestrichelte Linie zeigt den Trend. Links: Jahresmittel; Mitte: für Dezember; rechts: für Juli.

\begin{tabular}{l|l}
\hline Wertebereich & Zustand \\
\hline 2,0 und mehr & extrem feucht \\
\hline 1,5 bis 1,99 & sehr feucht \\
\hline 1,0 bis 1,49 & mäßig feucht \\
\hline$-0,99$ bis 0,99 & nahe normal \\
\hline$-1,49$ bis $-1,0$ & mäßig trocken \\
\hline$-1,99$ bis $-1,5$ & stark trocken \\
\hline-2 und weniger & extrem trocken \\
\hline
\end{tabular}

Tabelle 3 Wertebereich des SPIs mit dazugehörigen Kategorisierungen

Der SPI (Abb. 8) zeigt im Jahresmittel für alle Stationen einen vergleichbaren Verlauf, gibt jedoch an der Station Cottbus einen trockeneren Zustand an als an den anderen Stationen. Das ist im Juli und Dezember nicht so offensichtlich. Die Beobachtungen deuten über den gesamten Zeitraum einen positiven Trend an. Im Jahresmittel liegt er bei »normalen « Bedingungen, im Juli dagegen erkennt man, dass einzelne Jahre durchaus in die Kategorie »extrem trocken «bzw. »extrem feucht« fallen können. Im Dezember/Januar treten mehrere »stark trockene« Jahre auf, dagegen keine »sehr feuchten« Episoden.

Ein Vergleich der Modellergebnisse mit den Beobachtungen zeigt eine hohe Übereinstimmung der jährlichen Werte (nicht dargestellt). Dieses ermöglicht es, die Änderung des SPIs unter Einfluss der zwei gewählten Emissionsszenarien zu untersuchen. Man kann bei dem Zukunftsszenario RCP 4.5 keinen eindeutigen Trend des SPIs für die kommenden 100 


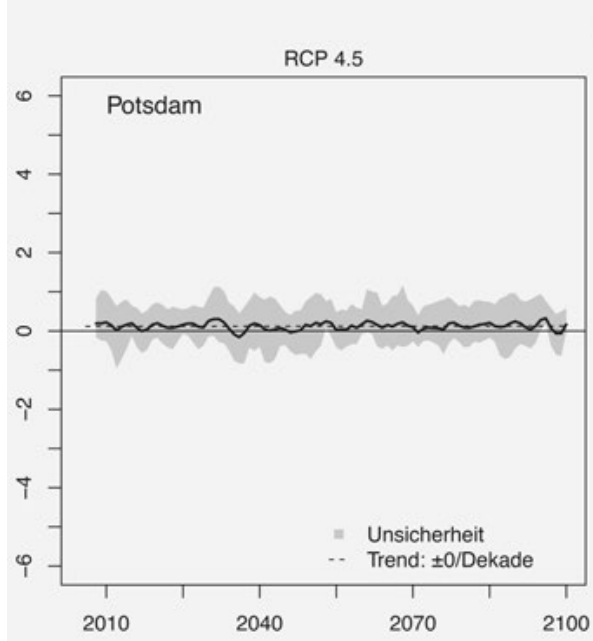

SPI

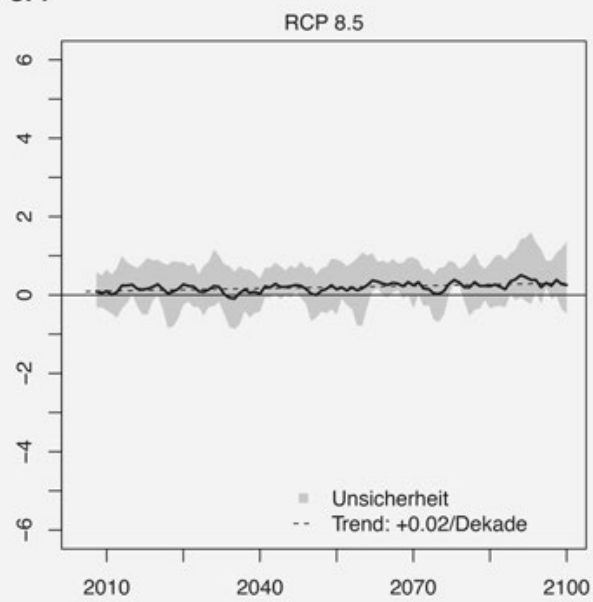

9 Modellprojektionen des jährlichen Mittels des SPI für Potsdam für den Zeitraum zwischen 2006 und 2100. Die grau schattierte Fläche beschreibt die Modellunsicherheit und die schwarze Linie das Mittel über alle Modellsimulationen. Die gestrichelte Linie zeigt den Trend. Links: Szenarium RCP 4.5, rechts: Szenarium RCP 8.5.

Jahre erkennen, für das RCP 8.5 Szenarium dagegen einen positiven Trend (Abb. 9), d.h. eine Zunahme der feuchten Phasen. Einer jahreszeitlichen Analyse kann man entnehmen, dass die feuchten Phasen im Winter vermehrt auftreten, während der Sommer unverändert bleibt.

Gerstengarbe et al. (2003) und Lotze-Campen et al. (2009) sagen mit statistischen Modellen, ausgehend von den Beobachtungen zwischen 1956 und 2005, einen insgesamt abnehmenden Trend der Jahresmittel-Niederschläge bis zum Jahr 2055 für die Region Berlin-Brandenburg vorher. Sie kommen zu dem Ergebnis, dass im Winter die feuchten Phasen zunehmen und im Sommer abnehmen werden.

\section{Zusammenfassung}

Der Klimawandel sorgt für globale Änderungen, die im regionalen Maßstab sowohl die Flora als auch die Fauna in den Parks Berlins und Brandenburgs beeinflussen werden. Um die Auswirkungen dieses Wandels für die Anlagen wissenschaftlich zu bestimmen, wurden die historischen Klimabedingungen im Zeitraum zwischen 1951 und 2005 durch eine Analyse der Beobachtungen untersucht und die zukünftigen Konditionen im Zeitraum zwischen 2006 und 2100 anhand von Modellsimulationen betrachtet. Der Vergleich von Beobachtungsdaten und Modellergebnissen ermöglicht die Bewertung der Zuverlässigkeit der Modellsimulationen. Um die Klimaveränderungen als Funktion der Treibhausgaskonzen- 
trationen in der Atmosphäre zu prognostizieren, wurden multiple Modellsimulationen für zwei Emissionsszenarien (RCP 4.5 und RCP 8.5) gewählt und ihre Ergebnisse analysiert.

Die Untersuchung der Mitteltemperatur zeigt, dass an den verschiedenen Standorten vergleichbare Temperaturen und Trends vorherrschen. Die Modelle können diese Entwicklung sowohl jährlich als auch saisonal zuverlässig wiedergeben. Im historischen Zeitraum (1956-2105) liegt eine mittlere Erwärmungsrate von $0,3^{\circ} \mathrm{C} /$ Dekade vor. Für die Zukunft sagen die Modelle eine Erwärmung von ca. $1^{\circ} \mathrm{C}$ für Szenarium RCP 4.5 und von ca. $3^{\circ} \mathrm{C}$ für Szenarium RCP 8.5 bis zum Jahr 2100 voraus. Analog findet man auch einen Anstieg der Maximum- und Minimumtemperatur. In beiden Fällen setzt sich die beobachtete Temperaturzunahme der Vergangenheit in der Zukunft weiter fort. Auch die abnehmende Anzahl von Tagen mit einer Minimumtemperatur unter $-5^{\circ} \mathrm{C}$ spiegelt den Erwärmungstrend wider. Die Anzahl der Sommer- und Hitzetage wird deutlich zunehmen, die der Frost- und Eistage dagegen abnehmen.

Der Niederschlag zeigt in den Beobachtungen einen leichten positiven Trend, der im Sommer stärker ausgeprägt ist als im Winter. Der normalisierte Niederschlagsindex SPI zeigt für den historischen Zeitraum keinen eindeutigen Trend. Im moderaten Szenarium (RCP 4.5) erkennt man auch für die Zukunft keinen eindeutigen Trend, für das progressive Szenarium sieht man eine Zunahme der Feuchtereignisse im Winter.

Um umfangreichere und pflanzenspezifische Ergebnisse zu erhalten, könnte man in zukünftigen Untersuchungen räumlich höher auflösende Modelle und pflanzenspezifische Prozess-Modelle einbeziehen. Klimabedingte Veränderungen im Bereich der Pflanzen können durch Einbeziehung phänologischer Daten, die sowohl regional als auch europaweit vorhanden sind (Dierenbach et al. 2013), näher analysiert werden.

\section{Danksagung}

Diese Forschung basiert auf einer Expertise, die für die Berlin-Brandenburgische Akademie der Wissenschaften verfasst wurde. Wir möchten den Arbeitsgruppen der verwendeten Klimasimulationen danken (MPI, KNMI, DMI). Ein weiterer Dank gilt der Earth System Grid Federation (ESGF), dem European Network for Earth System Modeling (ENES) und anderen Partnern der Global Organisation for Earth System Science Portals (GO-ESSP). Bianca Wentzel leistete technische Unterstützung bei der Auswertung der Daten.

\section{Literaturverzeichnis}

Albritton, Daniel. L.; Barker, Terry; Bashmakov, Igor A., Canziani, Oswaldo, Christ, Renate; Cubasch, Ulrich; Davidson, Ogunlade; Gitay, Habiba; Griggs, David; Houghton, John; House, Joanna; Kundzewicz, Zbigniew; Lal, Murari; Leary, Neil; Magadza, Christopher; McCarthy, James J.; Mitchell, John F. B.; Moreira, Rose Roberto; Munasinghe, Mohan; Noble, Ian; Pachuri, Rajendra; Pittock, Barry; Prather, Michael; Richels, Richard G.; Robinson, John B.; Sathaye, Jajant; Schneider, Stephen; Scholes, Robert; Stocker, Tho- 
mas; Sundararaman, Narasinham; Swart, Rob; Taniguchi, Tomihiro; Zhou, D. (2001): Climate Change 2001: Synthesis Report. Hg. von Robert T. Watson. Cambridge: Cambridge University Press.

Berliner Klimafibel $\left(2016^{2}\right)$ : 100 Jahre Wetteraufzeichnungen. Berliner Wetterkarte e.V. www.berliner-wetterkarte.de (07.01.2019).

Cubasch, Ulrich; Kadow, Christopher (2011a): Temperaturaufzeichnungen in Berlin für die letzten 310 Jahre. In: Globaler Wandel und Regionale Entwicklung - Anpassungsstrategien in der Region Berlin-Brandenburg. Hg. von Reinhard F. Hüttl, Rolf Emmermann, Sonja Germer, Matthias Naumann und Oliver Bens. Heidelberg: Springer, 30-36.

Cubasch, Ulrich; Kadow, Christopher (2011b): Global Climate Change and Aspects of Regional Climate Change in the Berlin-Brandenburg-Region. In: DIE ERDE 142, 2-20.

Dierenbach, Jonas; Badeck, Franz-W.; Schaber, Jörg (2013): The plant phenological online database (PPODB): an online database for long-term phenological data. In: International Journal of Biometeorology 57.5, 805812. 10.1007/s00484-013-06 (01.10.2018).

Gerstengarbe, Friedrich-Wilhelm; Badeck, Franz W.; Hattermann, Fred. F. F.; Krysanov,, Valentina; Lahmer, Werner; Lasch, Petra; Stock, Manfred; Suckow, Felicitas; Wechsung, Frank; Werner, Peter C. (2003): PIK Report No. 83: Studie zur klimatischen Entwicklung im Land Brandenburg bis 2055 und deren Auswirkungen auf den Wasserhaushalt, die Frost- und Landwirtschaft sowie die Ableitung erster Perspektiven. https:// www.pik-potsdam.de/4c/web_4c/publications/pik_report_83.pdf (01.10.2018).

Hayes, Michael J.; Lowrey, Jessica (2007): Drought Indices. In: Intermountain West Climate Summary 3.6, 2-6.

Hupfer, Peter; Chiemelwski, Frank-M. (1990): Das Klima von Berlin. Berlin: Akademie-Verlag.

Hupfer, Peter; Becker, Paul; Börngen, Michael (2013): 20000 Jahre Berliner Luft, Klimaschwankungen im Berliner Raum. Leipzig: Edition am Gutenbergplatz.

IPCC (2014): Klimaänderung 2014. Synthesebericht. Beitrag der Arbeitsgruppen I .II und III zum fünften Sachstandsbericht des Zwischenstaatlichen Ausschusses für Klimaänderungen (IPCC). IPCC, Genf, Schweiz. Deutsche Übersetzung durch Deutsche IPCC-Koordinierungsstelle, Bonn 2016.

Jacob, Daniela; Göttel, Holger; Kotlarski, Sven; Lorenz, Philip; Steck, Kevin (2008): Klimaauswirkungen und Anpassung in Deutschland - Phase 1: Erstellung regionaler Klimaszenarien für Deutschland. Abschlussbericht zum UFOPLAN-Vorhaben 20441 13. Dessau: UBA.

Lotze-Campen, Hermann; Claussen, Lars; Dosch, Axel; Noleppa, Steffen; Rock, Joachim; Schuler, Johannes; Uckert, Götz (2009): Klimawandel und Kulturlandschaft Berlin. PIK Report 113. Potsdam. www.pikpotsdam.de/research/publications/pikreports/.files/pr113 (01.10.2018).

Stocker, Thomas F.; Qin, Dahe; Plattner, Gian-Kasper; Tignor, Melinda; Allen, Simon K.; Boschung, Judith; Nauels, Alexander; Xia, Yu; Bex, Vincent; Midgley, Pauline M. (Hg.) (2013): Climate Change 2013: The Physical Science Basis. Contribution of working Group I to the 5th Assessment Report of the Intergovernmental Panel on Climate Change. Cambridge: Cambridge University Press.

\section{Datenquellen}

DWD Zugang zum Datenserver: https://www.dwd.de/DE/klimaumwelt/cdc/cdcl_node.html

Es wurden folgende Modellexperimente ausgewertet:

- EUR-11 \ICHEC-EC-EARTH\_(historical/rcp45/rcp85)\_r3i1p1\_DMI-HIRHAM5\_v1

- EUR-11\_ICHEC-EC-EARTH\_(historical/rcp45/rcp85) \_r1i1p1\_KNMI-RACMO22E\_v1

- EUR-11\MPI-M-MPI-ESM-LR\(histrocial/rcp45/rcp85)\_r2ilp1\_MPI-CSC-REMO2009\_v1

\section{Bildnachweis}

1-9 Emmanuele Russo, Ulrich Cubasch und Gregor Pittke. 